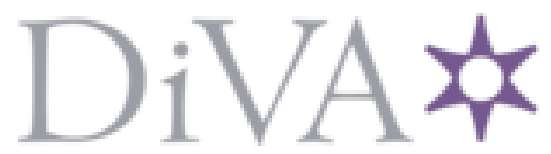

http://www.diva-portal.org

This is the published version of a paper presented at 15th International Conference on Advanced Learning Technologies (ICALT 2015), Hualien, Taiwan, 6-9 July 2015.

Citation for the original published paper:

Zbick, J., Nake, I., Milrad, M., Jansen, M. (2015)

A web-based framework to design and deploy mobile learning activities: Evaluating its usability, learnability and acceptance.

In: 2015 IEEE 15th International Conference on Advanced Learning Technologies (ICALT) (pp. 88-92). IEEE Press

http://dx.doi.org/10.1109/ICALT.2015.97

N.B. When citing this work, cite the original published paper.

(C) () 2015 IEEE. Personal use of this material is permitted. Permission from IEEE must be obtained for all other uses, in any current or future media, including reprinting/republishing this material for advertising or promotional purposes, creating new collective works, for resale or redistribution to servers or lists, or reuse of any copyrighted component of this work in other works.

Permanent link to this version:

http://urn.kb.se/resolve?urn=urn:nbn:se:Inu:diva-45518 


\title{
A web-based framework to design and deploy mobile learning activities: Evaluating its usability, learnability and acceptance
}

\author{
Janosch Zbick $^{1}$, Isabella Nake ${ }^{2}$, Marcelo Milrad ${ }^{1}$, Marc Jansen ${ }^{1}$ \\ Department of Media Technology \\ Linnaeus University, Sweden \\ ${ }^{1}\{$ janosch.zbick, marcelo.milrad, marcbjorn.jansen\}@lnu.se \\ ${ }^{2}$ isabella.nake@gmail.com
}

\begin{abstract}
This paper discusses the efforts carried out related to the design and development of a web-based framework that allows designing, deploying and executing mobile data collecting applications. Furthermore, it also allows analyzing and presenting the data that is generated during the mentioned process. The fact that the framework is completely web-based provides a platform independent execution of the mobile application on any mobile device with a web-browser. As a result that the whole life-cycle of creating, executing and discussing a mobile learning activity is implemented in pure web-based manner separates this work from similar efforts. In the course of this work, the current state of development of two of the components, the authoring tool and the mobile application is presented. This framework was introduced to teachers in an activity to follow up an initial study. On the basis of a workshop with teachers, we performed an explorative study regarding the technology acceptance and usability of two components of the proposed framework. The results are discussed and analyzed in this paper.
\end{abstract}

web-based framework; mobile learning; authoring tool; usability; technology acceptance

\section{INTRODUCTION}

Mobile devices, including smartphones and tablets are a common tool in educational environments [1]. In fact, in some countries a change in the curriculum occurred that encourages the usage of a wide range of information technologies to support teaching and learning [2]. The immense availability of smartphones and other mobile devices like tablets among students offers opportunities to integrate them into teaching practices. An important factor that researchers and developers need to consider is the high fragmentation of the market regarding mobile devices and their operating systems. Thus, to integrate students' mobile devices into educational settings, platform independent solutions are desirable.

Studies show that students' motivation can be stimulated if authenticity is introduced in the different learning tasks within an activity [3]. To provide authenticity, ongoing topics in the society and the learners interests can be integrated into these tasks [4]. This combined with the wide availability of mobile devices among students can take an important role in the day-to-day life. Their use for performing learning activities can increase at least initially the motivation of the students.
The development of emerging web applications, especially relying on HTML5 and JavaScript, allows taking advantage of features of modern mobile devices [5]. Nowadays, it is possible to access internal sensors like camera, microphone or positioning sensors from within mobile web-browsers and developers as well as researchers are not limited to native applications to exploit the potential of modern mobile devices. Therefore, pure web-applications based on HTML5 and JavaScript that can be executed in web browsers of mobile devices are a promising approach to address the issue of platform independent mobile applications.

Another crucial issue that needs to be considered while integrating mobile technologies in educational settings is the fact that teachers usually do not poses the technical nor the programming skills to design mobile applications that fit their own requirements. Thus, an essential factor that needs to be addressed by researchers is to offer non-technical skilled users a possibility to easily design and deploy mobile applications. In order to address some of these challenges, we have designed and developed a web-based framework to support teachers in creating their own mobile applications. The results of these efforts and an evaluation of its usability, learnability and acceptance are presented in this paper.

The remaining of the paper will give an overview of related work conducted in the field of Technology Enhanced Learning (TEL) in order to motivate our research efforts. Afterwards, we describe the development efforts of our framework that allows teachers to design and deploy their own mobile applications with the purpose of performing learning tasks. This is followed by a description of a user study. In the discussion section, we will present our findings and future plans.

\section{MOTIVATION AND RELATED WORK}

As discussed before, major challenges in today's research are related to the huge fragmentation of the market and the fact that we need to provide non-technical skilled users access to the design of applications that fit their needs. To tackle these two challenges, we gathered information about requirements [6] and developed a web-based framework that allows easily designing, deploying and executing crossplatform mobile applications [7]. 
Despite previous research efforts conducted in this field, few of the projects have addressed the whole process chain starting from authoring or have just been investigating it from a purely web-based perspective. Mulholland et al. [8] present with their nQuire project a way of designing learning tasks. However, they do not provide a possibility to deploy their learning scenarios automatically as a mobile application. Hwang et al. are lacking a way for teachers to author their own scenarios [9] and are not addressing the challenge of cross-platform development but only offer an Android application. The same applies for the LETS GO project [10] which provides an Android application to collect data and a web-based visualization environment for presenting the collected data but again not an authoring environment. LETS GO does also not address the crossplatform development challenge. Giemza et al. offer in their LEMONADE project an authoring tool to deploy mobile applications [11]. However, LEMONADE only supports the Android platform and thus is also not tackling the platform independency issue mentioned above. Baloian et al. [12] argue that HTML5 has an enormous potential for executing learning scenarios with mobile devices. Nonetheless, they are focusing on learning scenarios but not on authoring, deployment or data-analysis. All these facts indicated that similar research in this area is conducted. Nevertheless, no research project aims to offer consistent support throughout the whole lifecycle to design, execute and analyze mobile supported learning activities with a cross-platform solution.

Derived from the ideas discussed above, we have identified two main research questions that guide the efforts described in this paper. They are formulated as followed:

- RQ1: Is the proposed framework a fitting solution for teachers to design and deploy mobile applications?

- RQ2: Is a web-based solution fulfilling the requirements of mobile data collection applications?

\section{MLEARN4WEB}

mLearn4web is a framework, which allows teachers to design their own mobile learning applications, to use them with their students in educational settings, and to discuss the results afterwards. A field trip is one example of educational activities, which can be enhanced through technological support. The three components, authoring tool, mobile application and visualization of results, correspond to the phases of a field trip according to Krepel and DuVall [13], namely the pre-trip phase, the trip itself and the post-trip phase. During the pre-trip phase, teachers can create a mobile application which fits their needs and thus, eases the data collection during the actual field trip. In that phase, the students use the application on their mobile devices. Thereby, they can make use of the sensors integrated in the mobile devices, such as the camera, microphone, or geolocation. In the post-trip phase, the visualization tool allows the teacher to discuss the collected data and the results of the field trip with their students.
To make mLearn4web easily accessible for the users and not force them to download and install any application, it is built as a web-based tool that can simply be accessed by an Internet browser. The complete framework is built on HTML5 and JavaScript and consists of a number of components including an authoring tool, a mobile application generator and a visualization tool [6]. In addition, the backend is based on NodeJS. Thus, JavaScript is used on all layers and therefore, the data transfer is conducted using JSON objects. Furthermore, this goes well together with the MongoDB database, also directly handling JSON documents. Also, the characteristics of a non-relational database are perfect to handle semi- or unstructured data and its good scaling performance makes this choice a good fit for our solution.

\section{A. Authoring}

Using the authoring tool, teachers can design mobile applications. They can create multiple screens, each of them showing the data, which will later be displayed on one mobile screen. The tool offers various modules, which can be selected by the teachers and via "drag and drop", added to a screen. Those modules support mainly data collection, including making use of the built-in sensors of mobile devices. Teachers can choose between the following modules: "instructions", "textarea", "image", "video", "sound", "multiple choice", "numerical", "date" and "location". For each module, the teachers can add a short introduction. In comparison to the previous version of the tool as described in [6], the instruction module was added to provide teachers the possibility to give the students a task which does not require any data to be collected. Furthermore, the module "textinput" was removed, since a user study showed that the difference between "textarea" and "textinput" is not clear to the teachers [7]. Although this differentiation is well known among computer scientists, it seems to be irrelevant for non-technicians. This shows once more the importance for such tools to use formulations which are understandable for the users, in this case the teachers, and to avoid technical terms. Once the application design is completed, it can be saved to be executed on a mobile device. Figure 1 illustrates the authoring tool.

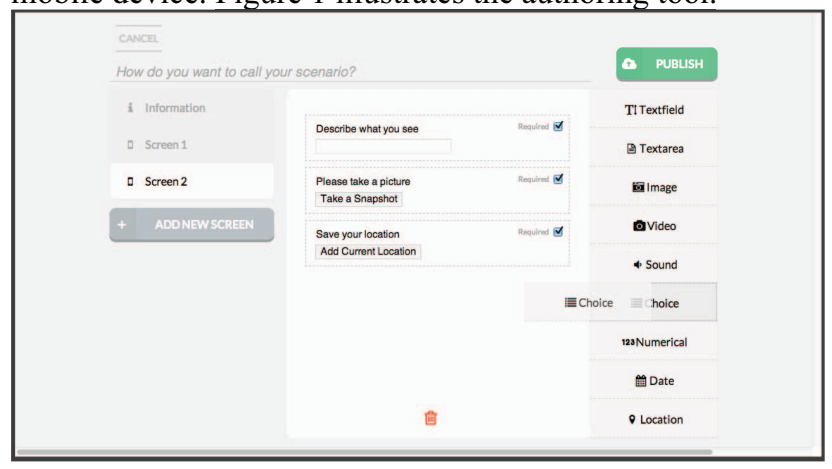

Figure 1. Screenshot of the authoring tool 


\section{B. Mobile Application}

When entering the mobile application, first a list with all available applications is shown, from which the user can select the desired one. Consequently, a description of the application is given and the user is asked to enter a group name, which can be useful for comparing and analyzing the results afterwards using the visualization tool. The following screens correspond to the screens designed with the authoring tool. Once the user is done with collecting all data, it can be submitted to the server to be stored into the database. Afterwards, the visualization tool can be used for analyzing the collected data. Figure 2 shows screenshots of the mobile application.

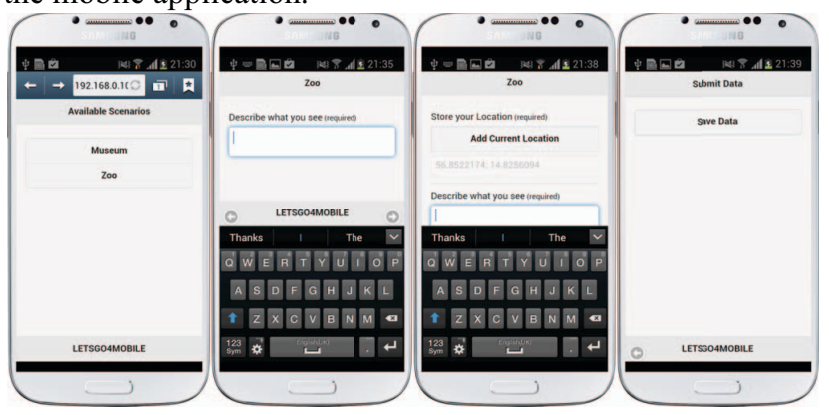

Figure 2. Screenshots of the mobile application

\section{Visualization}

This part of the framework provides a presentation of the collected data, giving the teachers the possibility to discuss the results of their learning activity with their students. Especially for collected geolocations, the visualization tool shows the data in a for the user more meaningful way. This means, the user is not presented with the geolocation coordinates, but gets them illustrated, e.g., on a map. Furthermore, modules located on the same screen are assumed to be related and thus, their visualizations show a connection as well. For example, if one screen of the mobile application contains the image module and the geolocation module, the visualization tool can present the images on a map according to the geolocations. At the current time, the visualization tool is not fully implemented yet and was thus not part of the user study.

\section{EVALUATION}

To investigate the research questions and analyze whether the proposed framework is a user-friendly solution to design and deploy mobile applications for educational purposes, a study with teachers was conducted. The study took place in two sessions, spread over two days with in total thirteen teachers. Each session had a duration of two hours and began with a short presentation of the authoring tool and its functionalities. The participating teachers were from the fields of sport, biology, geography and languages and had no training in IT. After the presentation, we designed an example scenario of an excursion to a zoo to provide a better understanding of the authoring tool. Finally, the workflow of the deployment was presented and the teachers were shown how to access the designed scenarios by executing the mobile application for the "zoo" scenario example. This was followed by letting the teachers design their own scenarios and perform test runs of their created scenarios on mobile devices. To answer our research questions, the teachers were given questionnaires which allowed us to collect some general information about the participants of the study as well as data regarding the technological acceptance and the usability. All questions with the exception of the question concerning the teaching experience were rated in a 7-level Likert scale [14] where 7 was labeled "Agree" and 1 was labeled "Disagree".

The thirteen teachers that took part in the study had an average of 16 years of teaching experience and thus we claim that our outcomes have a high level of relevance. We also gathered information about the background the teachers had in performing field trips and the technical support while organizing and performing field trips. The questionnaire with the general questions is presented in Table 1.

TABLE 1. GENERAL QUESTIONS

Q1: How many years of teaching experience do you have?

Q2: As a teacher, I think field trips are a valuable method to potentially enhance the learning experience.

Q3: I use technical tools to plan field trips.

Q4: I am taking advantage of mobile devices during a field trip.

Q5: If I use technical tools to support the planning of a field trip, those tools also offer support for the execution of field trips.

Findings of this questionnaire confirmed the outcomes of the study mentioned before, meaning that teachers are considering field trips as a valuable addition to traditional teaching methods. In our study the average of Q2 calculates to: $\mathrm{M}=6.38$. However, they do not take advantage of technological support to plan or perform field trips. The question regarding the technical support during the design process (Q3), calculates to a low average of $\mathrm{M}=2.54$ and the question about technical support during the field trip with mobile devices (Q4) calculates to a similar low average of M $=2.62$. During informal interviews with the participants, we learned that this is mainly because of the lack of software solutions and the fact that if software is available, it is often too complex to use and teachers get de-motivated by that. We conducted an initial study where we posed the same questions to the participants and the result was similar to the one of this study [7].

Additional to the general questions, the participants were asked questions derived from Davis' Technology Acceptance Model (TAM) [15]. TAM provides a method to identify the Perceived Usefulness (PU) and the Perceived Ease of Use (PEU). The questions used in this study were modified to adapt the questionnaire to our special requirements. The utilized questions about the PU of the mobile device and the authoring tool are presented in Table 2 and Table 3.

TABLE 2 QUESTIONNAIRE FOR PU OF THE AUTHORING TOOL

\begin{tabular}{l} 
The authoring tool ... \\
\hline Q1:... allows me to transform my ideas into tasks. \\
Q2: ... hinders me deploying mobile applications without \\
programming skills. $*$
\end{tabular}


Q3: ... allows me to design learning tasks for mobile devices. Q4: ... enables the design of data collection for mobile devices with internal sensors.

Q5: ... makes it difficult to deploy my scenario as a mobile application.*

*reverse question

TABLE 3. QUESTIONNAIRE FOR THE PU OF THE MOBILE APPLICATION

The mobile application ...

Q1: ... allows me to collect data with internal sensors.

Q2: ... hinders me entering text or numbers.*

Q3: ... allows me to execute tasks outside the classroom.

Q4: ... complicates the execution of learning tasks.*

Q5: Overall, I think such a mobile application is helpful to collect data.

Q6: ... helps me to better understand a learning topic.

*reverse question

The questionnaire about the PEU is the same for the authoring tool and the mobile application but has to be answered twice to differentiate between those two components. The questions can be found in Table 4. All used questionnaires are rated again in a 7-level Likert scale and the reverse questions are transformed accordingly for calculations

TABLE 4. QUESTIONNAIRE FOR THE PEU

\section{The mobile application ...}

Q1:... has a functionality that is easy to understand.

Q2: ... has not a user-friendly interface design.*

Q3:... has a good description of its functionalities.

Q4: ... has interaction methods that are not familiar to me.*

Q5: ... performs as expected.

Q6: ... is exhausting to use.*

*reverse question

To proof the reliability of the answers we calculated the Cronbach's alpha for the quesionnaires and it calculates to .746. However, we are aware of the problem that studies show that calculating the Cronbach's Alpha needs a bigger sample size than the one in this study [16]. Thus, we cannot provide a sincere test for reliability.

To get information about the technological acceptance, we considered the values of the PU and PEU. The overall average of the technological acceptance calculates to $M=$ $5.1808(S D=1.4771)$ where the authoring tool average calculates to $M=5.1564(S D=1.4334)$ with the PU of $M=$ $5.2615(S D=1.4511)$ and the PEU of $M=5.0513(S D=$ 1.4158). The average of mobile application calculates to $M=$ $5.2051(S D=1.5209)$ with the PU of $\mathrm{M}=5.3077(\mathrm{SD}=$ $1.554)$ and the PEU of $\mathrm{M}=5.1026(\mathrm{SD}=1.4878)$. Figure 3 shows a chart of the mentioned data.

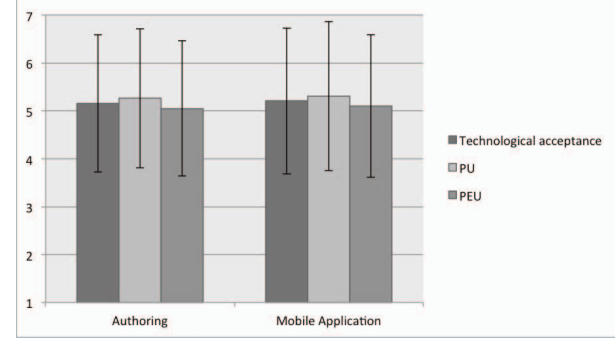

Figure 3. Overview of the technological acceptance results
These high values show that there is a high acceptance of the used web-technologies among the participants of the study. It also shows that the participants were able to design and execute a mobile learning scenario, although none of the teachers had a technical background. Thus, this data provide some initial evidence for elaborating an answer to RQ2 and we can claim that the technology that was used meets the demands of teachers of easy to use software.

The presented study is the second study where we tested against the technological acceptance derived from the TAM. However, we decided to not compare the first study presented in [7] with the actual one since too many variables changed. Both components, the authoring tool and the mobile application went through some changes and this study had also different participants than the one performed earlier. Therefore, we are not comparing the results of the two studies here. Nonetheless, we were pleased that the outcomes did not differ much from each other.

To gain knowledge about the usability of the proposed framework, we also asked the participants of this study to answer the questionnaire regarding the System Usability Scale (SUS) [17]. SUS is a questionnaire with ten questions for measuring the usability of software or hardware and is divided by measuring not only the usability but also the learnability of a system. Therefore, it is an important addition to our evaluation since we learned from the informal discussions that teachers need tools that are easy learnable. SUS is described as a low-cost usability scale that can be used for global assessment of systems' usability. SUS scores a system on a scale between 0 and 100. Two well-known approaches to rate the SUS-score are the ones developed by Bangor et al. [18] and Sauro et al. [19]. Both approaches relate the SUS score to the well known academic grading from A-F to provide a better understanding. For the presented system, the SUS-score is calculated to $\mathrm{S}=73.9744$ with a learnability score of $\mathrm{L}=79.5962$ and a usability of $\mathrm{U}$ $=72.5962$. According to grade-scales by Bangor et al. [18] and Sauro et al. [19] our system is graded C respectively BAdditional to the academic grading system, Bangor et al. [18] also added an adjective rating scale to the SUS-score where C refers to "good". Table 5 clarifies this and Figure 4 illustrates the data.

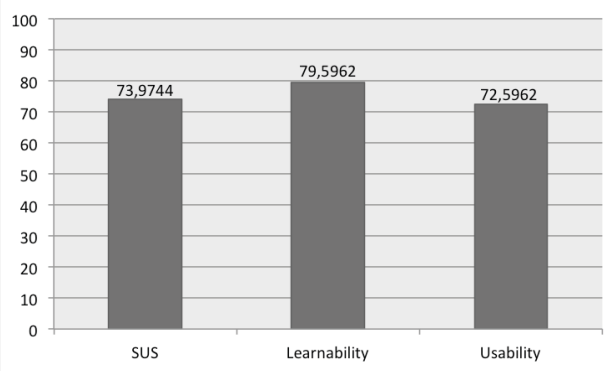

Figure 4. SUS-score with Learnability and Usability

\begin{tabular}{l}
\multicolumn{2}{|c|}{ TABLE 5. SUS-SCORE ACCORDING TO BANGOR AND SAURO } \\
\begin{tabular}{|l|l|cc|cc|}
\hline System & SUS-score & \multicolumn{2}{|c|}{ Bangor } & \multicolumn{2}{c|}{ Sauro } \\
& & Range & Grade & Range & Grade \\
\hline mLearn4web & 73.9744 & $70-80$ & C & $72.6-74$ & B- \\
\hline
\end{tabular}
\end{tabular}


Based on the good score of the investigated system, we have an even stronger indication that our system fits the requirements to be used by users with no technical background in order to design their own mobile applications and therefore provide answers to the RQ1. Especially highlighted can be the high value in learnability $(\mathrm{L}=$ 79.5962) here, which strengthens the argument that especially the non-technical users have a high acceptance using the presented framework.

\section{DISCUSSION AND FUTURE WORK}

This paper describes the efforts that are underway in developing a web-based framework that offers support throughout the lifecycles of designing a learning scenario.. The two components that are already implemented, the authoring tool and the mobile application are presented and a study with teachers that tries to evaluate the usability and the technological acceptance of those two components is described. By analyzing the outcome of the study, we can claim that the system has a high acceptance when it comes to the used technologies and is also easy to use and learn for non-technical skilled users. Thus, we are confident that the proposed web-based framework is a promising approach to offer support in the whole process of designing, executing and analyzing mobile learning activities.

The next step to enhance and complete the framework is the addition of a visualization tool to offer analytics to data that is generated during the activities. We are working on two approaches. One is an easy-to-use visualization tool that automatically generates visualizations based on the collected data. The second approach gives more experienced users the possibility to combine collected data sets and generate fitting data visualizations. In informal conversations during our studies and through experience we learned that it is advantageous to offer both, an easy-to-use approach for nontechnical user and an approach that offers more possibilities.

Furthermore, we keep enhancing our existing tools. During informal conversations with the teachers, we gathered information about potential enhancements like presenting data in the mobile device differently. E.g., at the time of the study saved geolocation data was represented by showing the coordinates, however it is advantageous for the user to visualize the coordinates on a map.

Although performance is not a crucial factor in the proposed framework, we are not satisfied with the performance of the mobile application. At the time of the study, the mobile application was implemented using jQuery Mobile. Currently we are re-developing the system using Ionic ${ }^{1}$ and AngularJS ${ }^{2}$ web application frameworks to enhance the performance.

\section{REFERENCES}

[1] S. Ruth, "Is E-Learning really working? The trillion-dollar question," Internet Comput. IEEE, vol. 14, no. 2, pp. 78-82, 2010
[2] The Swedish National Agency for Education (Skolverket), Curriculum for the compulsory school, preschool class and the leisure-time centre 2011. Stockholm: Skolverket, 2011.

[3] H. D. Mehlinger, "School reform in the information age," Phi Delta Kappan, vol. 77, pp. 400-407, 1996

[4] J. Lave and E. Wenger, Situated learning: Legitimate peripheral participation. Cambridge university press, 1991.

[5] N. D. Lane, E. Miluzzo, H. Lu, D. Peebles, T. Choudhury, and A. T. Campbell, "A survey of mobile phone sensing," Commun. Mag. IEEE, vol. 48, no. 9, pp. 140-150, 2010 .

[6] J. Zbick, M. Jansen, and M. Milrad, "Towards a web-based framework to support end-user programming of mobile learning activities," in 2014 IEEE 14th International Conference on Advanced Learning Technologies (ICALT), 2014, pp. 204-208.

[7] J. Zbick, I. Nake, M. Jansen, and M. Milrad, "mLearn4web: a webbased framework to design and deploy cross-platform mobile applications," in Proceedings of the 13th International Conference on Mobile and Ubiquitous Multimedia MUM '14, 2014, pp. 252-255.

[8] P. Mulholland, S. Anastopoulou, T. Collins, M. Feisst, M. Gaved, L. Kerawalla, M. Paxton, E. Scanlon, M. Sharples, and M. Wright, "nQuire: technological support for personal inquiry learning," IEEE Trans. Learn. Technol., vol. 5, no. 2, pp. 157-169, 2012.

[9] G.-J. Hwang, C.-C. Tsai, C. Y. Chen, and others, "A context-aware ubiquitous learning approach to conducting scientific inquiry activities in a science park," Australas. J. Educ. Technol., vol. 28, no. 5, pp. 931-947, 2012.

[10] B. Vogel, A. Kurti, M. Milrad, E. Johansson, and M. Müller, "Mobile Inquiry Learning in Sweden: Development Insights on Interoperability, Extensibility and Sustainability of the LETS GO Software System," Educ. Technol. Soc., vol. 4522, no. 17 (2), pp. $43-$ $57,2014$.

[11] A. Giemza, L. Bollen, M. Jansen, and H. U. Hoppe, "A flexible unified architecture to support heterogeneous multi-device learning environments," Int. J. Mob. Learn. Organ., vol. 7, no. 3, pp. 210-223, 2013.

[12] N. Baloian, J. Frez, E.-U. Diego, M. Jansen, and G. Zurita, "The Future Role of HTML5 in Mobile Situated Learning Scenarios," in 10th World Conference on Mobile and Contextual Learning (mLearn), 2011, pp. 249-257.

[13] W. J. Krepel and C. R. DuVall, Field Trips: A Guide for Planning and Conducting Educational Experiences. Analysis and Action Series. ERIC, 1981

[14] R. Likert, "A technique for the measurement of attitudes," Arch. Psychol., vol. 22, no. 140, pp. 1-55, 1932.

[15] F. D. Davis, R. P. Bagozzi, and P. R. Warshaw, "User acceptance of computer technology: a comparison of two theoretical models," Manage. Sci., vol. 35, no. 8, pp. 982-1003, 1989.

[16] L. S. Feldt and R. D. Ankenmann, "Determining sample size for a test of the equality of alpha coefficients when the number of part-tests is small.," Psychol. Methods, vol. 4, no. 4, pp. 366-377, 1999.

[17] J. Brooke, "SUS - A quick and dirty usability scale," Usability Eval. Ind., vol. 189, pp. 189-194, 1996.

[18] A. Bangor, P. Kortum, and J. Miller, "Determining what individual SUS scores mean: Adding an adjective rating scale," J. usability Stud., vol. 4, no. 3, pp. 114-123, 2009.

[19] J. Sauro and J. R. Lewis, Quantifying the user experience: Practical statistics for user research. Elsevier, 2012.

\footnotetext{
${ }^{1}$ http://ionicframework.com/

${ }^{2}$ https://angularjs.org/
} 